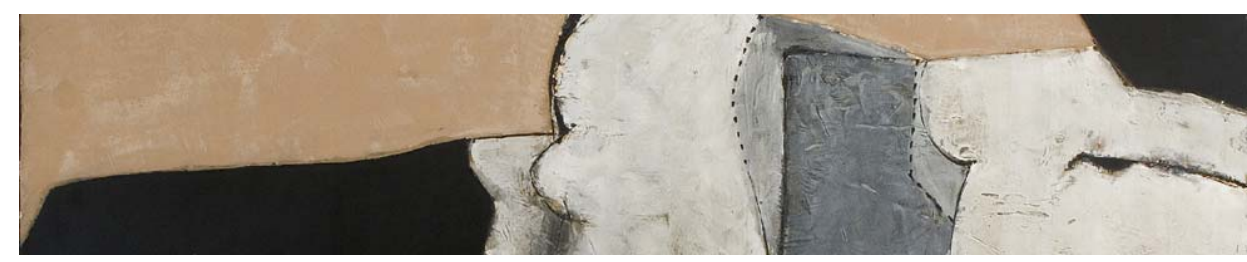

\title{
Consideraciones y propuestas de mejora de la comunicación en las instituciones públicas
}

\section{Considerations and Proposals in order to Improve Communication in Public Institutions}

\author{
José Antonio Alcoceba Hernando ${ }^{(*)}$ \\ Universidad Complutense de Madrid - España \\ alcoceba@ccinf.ucm.es \\ María Cristina Pinto Arboleda ${ }^{(*)}$ \\ Universidad Complutense de Madrid - España \\ mcpintoa@gmail.com
}

\section{Resumen}

Los resultados y conclusiones de dos investigaciones realizadas en torno al análisis mediacional del universo juvenil y su relación con la información institucional pública promovidas por el Instituto de la Juventud de España (Injuve), plantearon algunos interrogantes y consideraciones sobre el tratamiento de la información institucional sobre la juventud. En el presente trabajo se recogen dichas consideraciones y se ofrecen algunas reflexiones críticas sobre la producción comunicativa institucional, con el objeto de optimizar las posibilidades de

\begin{abstract}
The results and conclusions of two researches on the youth's image in the media and their relationship with the Spanish Instituto de la Juventud (Injuve) public information, raised some questions and considerations about the treatment of institutional information on youth issues. This article describes these considerations, as well as some critical reflections about the institutional production, in order to achieve visibility in the mass media concerning the key messages generated from public organizations.
\end{abstract}


visibilidad en los medios de los mensajes generados desde los organismos públicos.

Palabras clave: comunicación institucional pública, análisis mediacional de noticias, representaciones sociales, visibilidad en los medios, gabinetes de prensa.
Keywords: communication in public institutions, mediation analysis of news, social representations, media visibility, press offices. 


\section{O. INTRODUCCIÓN}

A partir del análisis mediacional de noticias ${ }^{1}$ de prensa, radio y televisión realizado por el Instituto de la Juventud de España (Injuve) en 2006 respecto a la imagen de los jóvenes en los medios de comunicación (Alcoceba y Mathieu, 2008²), se observaron algunas características específicas en el tratamiento mediático del universo juvenil. Una investigación posterior (Alcoceba Hernando, 2010 ${ }^{3}$ ), pretendió estudiar la producción institucional de información juvenil del Injuve (a partir de sus notas de prensa de los años 2005, 2006 y 2007) y su relación con las noticias que sobre juventud aparecieron, en esta ocasión tan sólo, en la prensa y en los medios digitales en ese periodo de tiempo.

Del tratamiento de la juventud y del análisis de la producción institucional y de su visibilidad en los medios, se observó cómo los discursos mediados contribuyen a la creación, refuerzo y transformación de las representaciones sociales. En palabras de Machado e Silva:

"Por medio de estos discursos provenientes de fuentes político-institucionales, los jóvenes son retratados en los medios de comunicación, que cumplen una función diseminadora de valores culturales, simbólicos y ideológicos cuando no solo transmiten, sino que preparan, elaboran y presentan una realidad que modifican y forman en el proceso de noticiarla" (Machado e Silva, 2004: 10).

Del resultado y conclusiones de las dos investigaciones citadas anteriormente, surgieron algunos interrogantes y consideraciones sobre el tratamiento de la información institucional sobre la juventud, que deter-

1 El análisis mediacional de noticias remite al estudio de los procesos de intervención de los medios de comunicación con respecto a los cambios que se producen en el entorno social. Trata de desentrañar las formas de producción institucionales en cuanto a la presencia, elaboración y difusión de los productos comunicativos en los medios.

2 El segundo de los trabajos de este manual incluye bajo el título "La imagen de los jóvenes en los medios de comunicación. Un análisis mediacional de las noticias sobre juventud publicadas en prensa, radio y televisión durante los meses de octubre y noviembre de 2006”, un análisis detallado de un conjunto de noticias sobre juventud seleccionadas en diversos medios de ámbito nacional, autonómico y local.

3 Este artículo recoge los resultados de una investigación (financiada por el Injuve) donde se analizaron las notas de prensa generadas por el Instituto de la Juventud durante los años 2005, 2006 y 2007 y las noticias relacionadas con dichas notas que aparecieron en la prensa y en los medios digitales en España en ese espacio de tiempo.

Mediaciones Sociales, № 9, II semestre 2011, pp. 111-133. ISSN electrónico: 1989-0494.

DOI: 10.5209/rev_MESO.2011.n9.38012 
minamos podrían ser extrapolables al tratamiento general de la comunicación institucional y de las notas de prensa en particular.

\section{LAS RELACIONES ENTRE COMUNICACIÓN INSTITUCIONAL Y CO- MUNICACIÓN MEDIADA A PROPÓSITO DE LA JUVENTUD}

Las instituciones políticas dirigen las sociedades en determinadas direcciones a través de las políticas públicas. Los mensajes sobre dichas políticas (a los que remite la comunicación institucional) deben mostrar las distintas dimensiones de los problemas sociales sobre los que tratan, transmitiendo al mismo tiempo la necesidad de intervención social sobre dichos problemas, generalmente asociada a la acción política de las instituciones.

Las relaciones informativas de la institución pública con los medios sirven para reforzar la relación institucional con los mismos y la consolidación de la imagen de dicha institución como fuente relevante de información sobre la temática juvenil. Pero dichas instituciones no siempre han entendido su servicio de comunicación como una necesidad de primer orden dentro de sus organigramas y cuerpos técnicos. Últimamente parece que sí se tiene en cuenta la importancia de estos departamentos, aunque generalmente cuentan con escasos recursos tanto materiales como de personal para llevar a cabo su cometido. La gestión de la sobrecarga de información sirve bien para ilustrar la dinámica de trabajo de la mayor parte de las organizaciones:

"Desde la llegada al despacho nada deja de ser input informativo: las llamadas que se reciben (de políticos, de unidades dependientes, de otros altos cargos de la institución, de otros gobiernos, etc.), la devolución de las llamadas, las reuniones a las que asiste, las preguntas del líder, los papeles que hay que contestar...." (Canel, 2007: 22).

Al mismo tiempo, la institución debe cuidar las relaciones con los medios y con los profesionales de los mismos. La cercanía y fluidez de estos contactos con los medios debe servir para reforzar la relación institucional y la imagen de la institución como fuente relevante de información sobre las problemáticas de las que se ocupan. Por esta razón, se debe planificar cada envío de notas de prensa a los medios y pensar en su verdadera justi-

Mediaciones Sociales, № 9, II semestre 2011, pp. 111-133. ISSN electrónico: 1989-0494.

DOI: 10.5209/rev_MESO.2011.n9.38012 
ficación. No siempre los mejores gabinetes de prensa son los que producen más llamadas a los medios. La política informativa de las instituciones públicas tiene una buena parte de negociación. Por eso, la institución debe identificar bien cuál es su capital y ponerlo "en juego" para lograr a cambio una buena cobertura. Como indican Berger y Luckman (1966: 93), "toda transmisión de significados institucionales entraña procedimientos de control y legitimización".

A este respecto, Pérez Curiel señala la importancia y los riesgos de estas relaciones:

"Es clave el papel que desempeñan los gabinetes o las oficinas de comunicación de los organismos oficiales que actúan como filtro para impedir el acceso directo de los medios de comunicación a las instituciones y, por otra parte, como centros emisores de información siempre interesada y con profundas cargas ideológicas, por lo que el medio deberá contextualizar siempre las informaciones que proceden de estas fuentes" (Pérez Curiel, 2008: 3).

Por tanto, el plan de comunicación que debe regir la elaboración de información institucional divulgada a través del gabinete de prensa, debe partir de un trabajo planificado y coordinado con los responsables de la política comunicativa de la organización, que tenga presente dichos principios básicos:

"El Plan General de Comunicación contrasta el grado de cumplimiento de los programas de comunicación, la eficiencia en el funcionamiento de los canales de comunicación interna y externa de la organización, así como resultado de los objetivos de comunicación de cada área o segmento" (Ramos, 2002: 53).

Del análisis de las notas de prensa realizadas por el Instituto de la Juventud de España entre 2005 y 2007 y de las noticias relacionadas con dichas notas en ese tiempo, destacamos aquí las consideraciones sobre las temáticas y los personajes políticos como elementos desde los que explicar las relaciones entre instituciones públicas y medios de comunicación.

1) El tratamiento de la juventud en las notas de prensa en el periodo mencionado estuvo dominado por una visión genérica y estereotipada: las menciones fueron casi siempre colectivas (los y las jóvenes en general, los jóvenes artistas, los jóvenes emprendedores, los jóvenes cooperantes, etc.).

Mediaciones Sociales, № 9, II semestre 2011, pp. 111-133. ISSN electrónico: 1989-0494.

DOI: 10.5209/rev_MESO.2011.n9.38012 
Junto a este tratamiento genérico de la juventud, aparece un buen número de comunicados de prensa que ni siquiera incluyeron menciones explícitas a los y las jóvenes; lo que refuerza el discurso abstracto donde dicho colectivo aparece tratado más como un objeto que como un grupo de personas concretas.

La normalización del discurso juvenil por parte de las instituciones, públicas en este caso, generó representaciones homogéneas que delimitan el concepto de la juventud y lo controlan, señalando los aspectos y objetivos en torno a los que se circunscribe su existencia. En este sentido apunta Revilla Castro (2001: 118): "cuando se habla de la juventud en general se tiende a referirse más a un discurso normativo que dice lo que es normal y lo que no lo es dentro de los jóvenes."

Cuando se hace referencia a los diferentes colectivos concretos de jóvenes, el tratamiento de dicha situación se centra en el apoyo y fomento institucional a través de programas y medidas (de cooperación, de investigación, empresariales, etc.), centrándose más en las posibles soluciones que en las causas y las problemáticas reales que las originan.

Este discurso, como afirma Revilla Castro (2001: 119), enfatiza las relaciones de poder, lo que refuerza una visión juvenil asociada a un estado de necesidad, que permite a las instituciones sociales (públicas especialmente) aparecer como responsables de las soluciones, lo que justifica la razón de su existencia.

2) Las temáticas de las notas de prensa. El tratamiento temático de la comunicación institucional del Injuve se relacionó especialmente con dos áreas de actuación político-administrativas sobre la juventud, adscritas, una al mundo sociopolítico (la emancipación) y la otra al ámbito cotidiano (el ocio y el tiempo libre).

Estas temáticas remiten a dos espacios juveniles considerados esenciales en los procesos de socialización modernos: la emancipación juvenil, que ha ido transformando sus trayectorias, convirtiéndolas en procesos más complejos e inciertos; y el ocio juvenil y la cultura, que se han consolidado como espacios claves e irrenunciables en la construcción de las identidades juveniles. 
El frecuente tratamiento de estas temáticas en las notas de prensa viene a reforzar la visión institucional sobre la juventud. El Instituto de la Juventud interviene comunicativamente resaltando la necesidad de su actuación política en ámbitos productivos como el de la emancipación juvenil, pero también sobre otros espacios de socialización y de construcción identitaria como los del ocio y la cultura juveniles:

"Para explicar las características del tratamiento temático en la prensa escrita, se parte del estudio de las rutinas periodísticas que consiste no sólo en la capacidad de seleccionar el tema, sino en una competencia de contextualización. Donde 'los medios de comunicación de masas, como institución pública, son piezas fundamentales para el equilibrio del sistema social' (Luhmann, 1997: 38). En tanto que establecen los temas de la agenda de discusión pública mediante una serie específica de political issue (en un trayecto que va de la exposición pública de temas controvertidos a la búsqueda de soluciones). Se infiere el potencial de los medios para decidir, inducidos por el poder o por sus propios intereses, qué, cómo y cuándo publicar qué temas, y cómo encausar mediáticamente sus efectos en la vida política" (Santillán, 2010: 2).

Este autor resalta como una buena oportunidad para los medios utilizar dichos temas propuestos desde las organizaciones políticas, para contextualizarlos y tratarlos en profundidad:

"En este caso, los periodistas mediante la información que publican contextualizan el tema político, entendido como una valoración periodística para reconocer los argumentos con que se debate en los medios, en palabras del profesor Lluís Badia (Badia, 1992: 210), caracterizada por el tratamiento a profundidad de los temas y problemas que se ejercen mediante la instrumentación de un conjunto de rutinas periodísticas y de estrategias editoriales" (Domínguez, 2005: 2).

Esta cuestión remite hacia la independencia de los medios más allá de sus líneas editoriales, para aprovechar los acontecimientos señalados desde las instituciones políticas, pero apuntando hacia la contextualización de los temas en torno a otros puntos de vista ideológicos distintos a los propuestos por dichos organismos.

3) En cuanto al tratamiento temático general de las noticias, es decir, la adscripción temática de los hechos seleccionados por los medios a partir de los comunicados institucionales; se refuerza una visión pragmática de la

Mediaciones Sociales, № 9, II semestre 2011, pp. 111-133. ISSN electrónico: 1989-0494.

DOI: 10.5209/rev_MESO.2011.n9.38012 
juventud asociada a su situación, generalmente desfavorecida, con respecto a temas como el empleo, la vivienda, las políticas públicas generales, o la investigación de problemas sociales. También aparecen noticias centradas en las necesidades de intervención destinadas a paliar las carencias juveniles en el ámbito cultural y del tiempo libre.

La institución aparece como interventora y redentora de las situaciones desfavorables concretas (tanto en lo productivo: empleo y vivienda) como en lo reproductivo (el ocio y la cultura).

El homogéneo tratamiento de los temas en las noticias y en los notas, pone de manifiesto el modelo mediador existente entre las instituciones y los medios. La selección de información proveniente de los organismos públicos es reelaborada siguiendo la orientación temática que propone dicha institución. Esto consolida el papel de las instituciones como fuentes fiables y confiables para los medios.

Teniendo en cuenta esta reproducción noticiosa de los puntos de vista ofrecidos por la comunicación institucional, se puede afirmar que los medios sirven como correas de transmisión de los mensajes políticos, no sólo en la selección de temas en torno a los que se centran los debates sociales, sino también en cuanto a las perspectivas ideológicas que se proponen.

La trascendencia ideológica de esta homogeneidad y por tanto de información desde otras perspectivas por parte de los medios sobre los temas y sus dimensiones ideológicas, entronca con los trabajos de Niklas Luhmann sobre la opinión pública como una:

"estructura temática de la comunicación pública, fundamentada en el hecho de que ante el número ilimitado de temas que pueden ser reflejados por la comunicación pública, la atención del público sólo puede manifestarse como limitada. La opinión pública deja de ser una generalización de las opiniones individuales para convertirse en instrumento de selección contingente de los temas de la comunicación política, al tiempo que reduce la complejidad social de los sistemas" (Domínguez, 2005: 3).

Esto supone una reducción de la libertad de las audiencias para pensar los temas desde perspectivas críticas más completas sobre las problemáticas sociales. Como apunta Machado e Silva (2004: 6), en este sentido "los medios construyen la realidad social, crean narrativas llenas de representa-

Mediaciones Sociales, № 9, II semestre 2011, pp. 111-133. ISSN electrónico: 1989-0494.

DOI: 10.5209/rev_MESO.2011.n9.38012 
ciones y las difunden como verdad absoluta. Las narrativas sociales que alimentan el imaginario son simplificadas y muchas veces estereotipadas".

4) Entre los objetivos del estudio de los personajes institucionales, el principal interés se centró en el tratamiento que se ofrece del Instituto de la Juventud, atendiendo al análisis de sus programas, servicios y representantes.

La labor del Injuve en estos tres años en cuanto al tratamiento informativo interno estuvo marcada por la actividad institucional principalmente asociada a la agenda de la Dirección General.

Esta conclusión evidencia el control que los responsables políticos ejercen en cuanto a los mensajes que se generan desde las instituciones, como afirma Van Dick:

"Estas élites controlan los discursos políticos, mediáticos, educativos, científicos, legales y burocráticos. El discurso público, como los reportajes de noticias, también se refiere más frecuentemente a ellos. Estas élites simbólicas pueden controlar no sólo los discursos en sus propios dominios -tal como los presidentes podrían controlar el discurso político y los docentes el discurso científico- sino también parte de los otros discursos públicos; por ejemplo a través de ruedas de prensa, entrevistas o talk shows. Además, dicho control podría ser visto de forma más concreta en la selección de temas y en las formas (frecuentemente positivas) en las que, estas élites son descritas y citadas" (Van Dijk, 2004: 10).

Esta personalización de los mensajes en torno a los personajes políticos no tiene por qué suponer una dinámica informativa necesariamente negativa, aunque sí es importante entender cuál debe ser el papel que juegan los responsables y las instituciones; en muchos casos, las organizaciones aparecen funcionalmente subordinadas a los responsables políticos y no al contrario como debiera suceder.

\section{CONSIDERACIONES Y PROPUESTAS DE MEJORA DE LA COMUNICA- CIÓN EN LAS ORGANIZACIONES PÚBLICAS}

El tratamiento de la juventud a partir del análisis de la producción institucional y de su visibilidad en los medios, requirió de una reflexión sobre cómo los discursos mediados contribuyen a la creación, refuerzo y

Mediaciones Sociales, № 9, II semestre 2011, pp. 111-133. ISSN electrónico: 1989-0494.

DOI: 10.5209/rev_MESO.2011.n9.38012 
transformación de las representaciones sociales. En palabras de Machado e Silva:

"Por medio de estos discursos provenientes de fuentes políticoinstitucionales, los jóvenes son retratados en los medios de comunicación, que cumplen una función diseminadora de valores culturales, simbólicos y ideológicos cuando no solo transmiten, sino que preparan, elaboran y presentan una realidad que modifican y forman en el proceso de noticiarla" (Machado e Silva, 2004: 10).

El mensaje es un reflejo de la estrategia que incorpora los objetivos que la institución se ha marcado. La comunicación política consiste en transmitir un punto de vista determinado sobre la realidad; las perspectivas de un conflicto o problema sociopolítico generalmente difieren según las implicaciones de los sujetos y sus representantes comunicativos.

\subsection{La comunicación interna y externa de las organizaciones: las mi- siones del gabinete de comunicación}

La ejecución de las políticas de comunicación institucionales suele realizarse a partir de gabinetes de prensa; departamentos que cada vez han adquirido una mayor relevancia en las estructuras organizativas, debido a la gran importancia que ha adquirido en nuestras sociedades la labor de informar y transmitir una imagen adecuada frente a la opinión pública: "El gabinete de prensa es hoy por hoy un instrumento imprescindible para conseguir que la imagen de una entidad o empresa llegue al gran público (...). A menor consideración de la institución o la empresa sobre la importancia del gabinete de prensa, menor será su penetración social” (Del Río Martínez, 2001: 131).

El gabinete de prensa supone, como señala Mateos Martín (2003), introducir en el trabajo un factor profesional especializado para gestionar las relaciones informativas. Dicha profesionalización debe regir los principios de los profesionales comunicativos que trabajan en los gabinetes en una triple dirección: 1) Transmitiendo contenidos informativos plurales y contrastados para que la opinión pública pueda valorar la calidad de sus representantes políticos; 2) Especializándose en un tratamiento de la información que tenga cabida entre los contenidos informativos de los medios; 3. Adaptando su trabajo comunicativo a las dinámicas que imponen los cam-

Mediaciones Sociales, № 9, II semestre 2011, pp. 111-133. ISSN electrónico: 1989-0494.

DOI: 10.5209/rev_MESO.2011.n9.38012 
bios sociales (uso de nuevos recursos y soportes, contacto directo con los medios, etc.).

De la misma forma que ocurre con el resto de organizaciones, la planificación de las labores de comunicación, cumple un papel fundamental para consolidar y transmitir los objetivos perseguidos por cada institución pública. La comunicación institucional pública que se elabora, debe ser transmitida a la opinión pública de forma que ésta, además de conocer el funcionamiento de la organización, entienda y asuma de forma positiva la labor que allí se realiza.

El primer paso para que esta meta sea alcanzada, consiste en coordinar una estructura de comunicación interna ajustada a los objetivos de la organización, que a su vez se transforme en un modelo de comunicación externa con una imagen de coherencia y consistencia que transmita confianza ante la ciudadanía:

"La estrategia de comunicación global debe tener en cuenta, por tanto, la información emanada de las distintas áreas de la organización, pero también debe integrar y armonizar la comunicación interna y la externa de la institución. Se trata de definir una imagen coherente que encaje con la finalidad de la organización y la potencie" (Camacho Markina, 2001: 5).

\section{Como afirma García Rivas:}

"Una organización utiliza la comunicación institucional como su propia expresión oficial ante la sociedad, mediante todos los medios de comunicación a su alcance (...) Y siempre con una doble finalidad. En primer lugar, facilita el funcionamiento interno de la propia organización, a través de lo que se denomina comunicación interna. Y en segundo lugar, favorece la creación de una determinada imagen pública de esa organización, por medio de su comunicación externa" (García Rivas, 2003: 4).

Todos los mensajes que se emiten en una organización, deben estar perfectamente coordinados por un plan estratégico de comunicación que responda a estos principios y perfile una imagen determinada de la institución ante la sociedad. Para ello, la comunicación interna debe estar totalmente integrada en la cultura corporativa de la organización, y la externa debe consolidar una imagen de confianza ante la ciudadanía. 
Dentro de los modelos de comunicación interna desarrollados con éxito en algunas instituciones públicas, se destacan mecanismos como:

- Identificar, establecer y mantener los objetivos de comunicación que en el interior de la organización se deben desarrollar para tratar de conseguir que todas las funciones estén de acuerdo a la imagen corporativa a transmitir. "Las instituciones públicas necesitan trabajar por la identidad corporativa (...) es necesaria la implementación interna de la identidad a través de la cultura y filosofía corporativa y la correcta difusión externa de esa identidad para provocar un clima favorable" (Cancelo San Martín, 2007: 74).

- La persona designada por la institución para expresar ante la opinión pública los mensajes, debe estar capacitada para ejercer de portavoz. Su conocimiento sobre la organización, así como sus habilidades comunicativas son determinantes para que los mensajes se transmitan de forma clara y coherente, y respondan a las necesidades de los medios.

Por su parte, en la planificación de la comunicación externa se debe prestar atención a cuestiones como:

1) Trabajar para difundir la actividad de la institución en los diferentes medios de comunicación (del ámbito nacional, regional y local), teniendo siempre presente el tipo y calidad de mensajes (transparencia, veracidad y rapidez) que se quiere hacer llegar a la opinión pública. Y al mismo tiempo, como sostiene Coldwell (2001: 8), la comunicación institucional debe garantizar las más altas normas de profesionalismo y conducta ética. "Con transparencia y rigor, (...) la comunicación externa es capaz de transmitir a sus destinatarios la imagen y los principios y funciones de su organización, pero también, de situar socialmente a su organización, de manera que sea reconocida por la opinión pública en general” (García Rivas, 2003: 4).

2) Reforzar la relación con los medios y periodistas, valorando su importancia como orientadores de la opinión pública. También es importante señalar que se deben optimizar y planificar las estrategias de comunicación. Aunque ninguna organización política niega la importancia de las relaciones con los medios, como afirma Xifrá Triadu (2003), sin embargo es relativamente frecuente limitar dichas relaciones a una relación esporádica y testimonial, que no atrae la atención de los profesionales de la comunicación.

Mediaciones Sociales, № 9, II semestre 2011, pp. 111-133. ISSN electrónico: 1989-0494.

DOI: 10.5209/rev_MESO.2011.n9.38012 
3) Se debe desarrollar un trabajo de seguimiento respecto a la calidad e incidencia informativa de las acciones gestionadas por el gabinete en los diferentes medios de comunicación. Presencia/ausencia de referencias a las notas, tratamiento de los temas y de los personajes de acuerdo a los enfoques previstos, etc.

Dicho trabajo de seguimiento debe tener en cuenta información relevante para la institución. En la investigación de las notas de prensa del Instituto de la juventud, se observó que la repercusión mediática de las mismas, tuvo una mayor repercusión en los medios digitales ( $2 / 3$ de noticias) que en la prensa ( $1 / 3$ de noticias). Este hecho sirve para considerar que los medios digitales, van ganando audiencia entre las nuevas generaciones juveniles, más familiarizadas con estas nuevas vías alternativas a la comunicación pública tradicional, representada por las noticias pertenecientes a medios impresos. Ante estos nuevos medios, también hay que tener presente el desconocimiento de las audiencias de los mismos y del tipo de consumo informativo que se hace de ellos. Si bien es cierto que van ganando espacio, no es menos cierto que en estas dinámicas comunicativas, la representación de la imagen institucional se construye sobre todo a partir de la producción informativa que elaboran los medios tradicionales.

También es importante tener en cuenta que los medios digitales están creando nuevas secciones especializadas, donde los contenidos juveniles encuentran un acomodo más flexible, incluso en secciones específicamente juveniles. A estos cambios en el tratamiento de la información juvenil, no debieran ser ajenas las instituciones públicas, a la hora de orientar, e incluso sugerir, la inclusión de nuevas secciones y apartados que respondan a las nuevas temáticas relacionadas con el ámbito juvenil.

De la misma manera dio la sensación de que los medios que tomaron en cuenta la información del Injuve, incorporaron dicha información en páginas secundarias y con poca cobertura. Esta apreciación debe tomarse en consideración para tratar de hacer un esfuerzo por llegar a prensa con mayor índice de divulgación.

4) Respecto al contenido de las noticias elaboradas a partir de las notas de prensa recibidas, se observó frecuentemente que los medios reproducen la información tomando extractos de las notas de forma literal. Este hecho, 
lleva a suponer que los medios elaboran sus productos comunicativos con la información que se les aporta. Por esto, es importante considerar que si además de la nota de prensa se les incluye un dossier informativo (con imágenes de apoyo, estadísticas, fuentes informativas), se brinda la oportunidad de elaborar productos que permitan visiones en profundidad de ciertos temas, debates o miradas críticas y reflexivas.

5) El objetivo de afianzar una comunicación bidireccional entre ciudadanos e instituciones públicas, parte de conocer el funcionamiento y rol de los medios para adaptar la información de la organización a los objetivos institucionales y mediáticos.

En este sentido, sería importante clarificar cuál es el tipo de público al que, según los objetivos concretos, se dirigen los mensajes; con la intención de crear/prever ciertas necesidades informativas entre los diferentes colectivos identificados como posibles audiencias. Es importante reconocer que para una institución existen diferentes grupos de público objetivo, y así identificar el tipo de información que ellos requieren. "Después de las relaciones con los medios, la función comunicativa externa más importante que puede llevar a cabo la administración es la comunicación con los ciudadanos" (Camacho Markina, 2001).

La estrategia comunicativa de las instituciones públicas se podría acotar en torno a los factores situacionales recogidos por Canel (2007) del modelo de contingencia estratégica de Garnett:

- Los objetivos de comunicación. Las organizaciones se rigen por principios generales que generalmente remiten a las políticas públicas en los ámbitos en los que se desenvuelven.

- El público al que se dirige la comunicación, que se diferencia en función del background, rol que juega en relación con la institución, intereses, necesidades, etc. La comunicación institucional debe tomar en cuenta tanto los intereses específicos de los profesionales de la comunicación, como los de los destinatarios finales, es decir, los ciudadanos y ciudadanas.

- Las estructuras de las instituciones públicas determinan sus dinámicas de trabajo informativo. En este sentido, es importante tomar en 
cuenta el papel que se concede a la comunicación dentro de las organizaciones (recursos humanos y materiales, funcionamiento de la comunicación interna, etc.). Una particularidad de estas organizaciones es que suelen estar sujetas a los ciclos políticos que determinan en buena medida la labor de comunicación externa.

- La persona que hace de comunicador: puesto que ocupa, y habilidades y credibilidad con que cuenta. El trabajo que se haga desde su gabinete de comunicación deberá dirigirse hacia la consolidación de la organización como fuente de información estable y confiable dentro del ámbito de actuación pública.

\subsection{Las notas de prensa}

Toda institución pública debe tratar de generar representaciones que incluyan puntos de análisis y reflexión entre la opinión pública a través de las notas de prensa que emite. Una vez sean recibidas por los medios, el objetivo se centra en lograr que las cuestiones importantes para ella sean incluidas en la agenda de medios.

Además de las notas de prensa, existen diversos recursos informativos que pueden ser utilizados como complemento, según el objetivo comunicativo que se persiga. Herramientas como el dossier de prensa, los contenidos audiovisuales de apoyo, o el suministro de fuentes estadísticas, son valoradas por los periodistas para crear productos de mayor incidencia informativa, tales como reportajes, entrevistas en profundidad, crónicas, foto-reportajes, etc.

En el conjunto de notas analizadas se identificaron tres modalidades informativas de notas de prensa, que sirven de ejemplo para observar cómo éstas pueden variar según los objetivos comunicativos que se persiguen:

1) Informativas sobre actividades internas del Injuve. Promoción de actividades internas sobre el funcionamiento de la propia institución o sobre programas y actividades promovidos por la misma. Estas notas ofrecieron información sobre el funcionamiento de la institución y de su actuación interna, que en este caso remitía a su presencia social, por tratarse de una institución política.

Mediaciones Sociales, № 9, II semestre 2011, pp. 111-133. ISSN electrónico: 1989-0494.

DOI: 10.5209/rev_MESO.2011.n9.38012 
2) Informativas sobre actividades externas en las que participa el Injuve. Se trató de actividades ajenas a la organización, en las que ésta participa con otras instituciones públicas y privadas, generalmente en aras de la implementación y desarrollo de políticas de juventud: participación en eventos y acontecimientos de otras o con otras instituciones.

3) Convocatorias de prensa. Aparecieron un número de notas que remitiendo a actividades internas o externas sirvieron para convocar a los medios a través de una rueda de prensa donde suele estar presente el Injuve a través de alguno de sus representantes, generalmente la Directora. En estos casos, la institución convocó a los periodistas para comunicarles algo personalmente, generalmente a través de un representante cualificado relacionado con la Dirección General.

De acuerdo con algunas investigaciones, parece probada la baja efectividad de las notas de prensa que se mandan a los medios de comunicación ${ }^{4}$. Las instituciones debieran analizar los porqués de esta escasa acogida por los medios, reflexionando sobre las necesidades y expectativas mediáticas, o sobre si su lenguaje responde o está redactado con mentalidad periodística.

\subsection{Principales retos para los comunicadores}

Ahora bien, éste que es un requerimiento para todo tipo de instituciones, en el caso de un organismo público, los responsables del gabinete de comunicación en su quehacer profesional se enfrentan a cuestiones cómo:

\footnotetext{
${ }^{4}$ Un estudio dirigido por Estudio de Comunicación y realizado por Demométrica, en colaboración con la Federación de Asociaciones de Periodistas de España (FAPE), la Asociación de Periodistas de Información Económica (APIE) y la Asociación Nacional de Informadores de Salud, concluyó con que los periodistas sólo consideran publicables el $12 \%$ de las notas que reciben, es decir, desechan el $85 \%$ de los comunicados de prensa de empresas e instituciones que llegan a las redacciones, y prestan más interés a las declaraciones que a los datos. Estas son algunas de las conclusiones que más llaman la atención del informe "Periodistas, Empresas e Instituciones, 2007”, en el que participaron 220 redactores jefe y jefes de sección de las secciones de economía, sociedad e información sanitaria de medios de comunicación de toda España (ANIS). Recuperado el 18 de diciembre de 2009 de http://www.elmundo.es/elmundo/2006/06/27/comunicacion/1151414416.html
} 


\section{- ¿Qué implicaciones tiene para una institución redactar con menta- lidad periodística?}

En el caso de la nota de prensa, entendida como un recurso de comunicación institucional, se requiere adaptar el mensaje a unas formas determinadas de producción comunicativa:

1) Ajustarse en la medida de lo posible, a los requerimientos propios de los medios informativos, lo que sugiere divulgar información que sea susceptible de convertirse en un hecho noticioso de interés para la ciudadanía. Del tratamiento que se le dé a la información en clave noticiosa, depende que sea considerada de interés público por parte de los periodistas. De esta forma, se facilita que algunos medios (por ejemplo los digitales), copien la información tal y como se les presenta en las notas de prensa.

Es importante tener presente que no es incompatible divulgar comunicación institucional pública que responda a los intereses de los medios.

2) Dentro del estilo periodístico, muchas veces la información relacionada con el funcionamiento interno de las instituciones resulta intrascendente e irrelevante. Sería recomendable recapacitar acerca del excesivo número de notas sobre la agenda de eventos, el quehacer diario de la institución o la participación de personajes institucionales internos y externos.

Limitarse a divulgar información de este tipo, puede tener un efecto contraproducente (como podría ser que los periodistas relacionen a la institución con este tipo de tratamiento informativo), y por el contrario, información relevante como las propias iniciativas, investigaciones o seguimiento de políticas, pierda su real espacio en los medios.

3) Respecto a los actos organizados para convocar a los medios, es importante tener en cuenta que la producción de la nota de prensa, puede resultar poco atrayente a los periodistas sino se justifica dentro del contexto de la presentación de iniciativas que aporten información relevante para construir la noticia. Se aconseja que las notas de prensa incluyan "ganchos informativos" que justifiquen el desplazamiento del periodista hacia el lugar de la convocatoria. Más allá de un acto oficial, el valor y trascendencia de una iniciativa debe ser el eje que estructure la nota de prensa. 


\section{- ${ }^{\text {LLos temas abordados importan a la opinión pública? }}$}

Como ocurre por lo general con diversas temáticas, la responsabilidad sobre lo que condiciona el interés informativo por parte de los medios, no recae exclusivamente en el tema, sino en la forma como son presentados a los medios y en los "ganchos informativos" empleados para justificar su publicación.

Dentro del lenguaje periodístico, se da prioridad a la información que es contrastada con datos que sirvan para producir un efecto de "llamada de atención" sobre determinadas temáticas.

Por ejemplo, si se difunde información relacionada con un asunto concreto, es una buena oportunidad para ofrecer cuestiones relacionadas con dicha temática. En muchos casos, esta información adicional facilita su utilización como recurso. Incluso se podrían utilizar nuevas posibilidades comunicativas de enlaces y recursos en red, como forma de facilitar y hacer más interesante la información a los medios.

La utilización de recursos gráficos (fotografías, gráficos estadísticos, imágenes, etc.), siempre son de gran utilidad para los medios impresos y digitales, ya que sirven de apoyo visual a la información y facilitan su lectura.

- ¿De qué forma deben ser tratados los temas para que a la vez de interesar a los medios, aporten en la construcción de la representación que interesa a la institución?

1) Una institución pública debe emitir diferentes tipos de mensajes. Según el objetivo de la comunicación, se hace necesario diferenciar el tipo de nota de prensa que para cada ocasión se deba elaborar. Es muy diferente la forma como se diseña una convocatoria de medios, la cual se debe caracterizar por incluir información concisa y precisa sobre el evento, a como se diseña una nota informativa sobre los resultados de una investigación o los objetivos de una iniciativa. En este último caso, es importante que la información además de ser clara e interesante; incluya los datos suficientes para que los periodistas se sientan motivados a indagar e iniciar un análisis sobre la temática de la que se quiere informar.

2) En cuanto al tratamiento de la información y la reinterpretación que los periodistas pueden hacer de los datos presentados en la nota de prensa,

Mediaciones Sociales, № 9, II semestre 2011, pp. 111-133. ISSN electrónico: 1989-0494.

DOI: 10.5209/rev_MESO.2011.n9.38012 
merece especial atención, que los datos suministrados sean claros y no se presten a malentendidos. Se debe hacer un esfuerzo para que los resultados y su valoración no sean utilizados por los medios de comunicación de masas para generar lecturas erróneas, que pueden perjudicar la imagen de un colectivo, una institución, sus representantes, etc.

3) En el caso de las notas de prensa analizadas, se observó que el número de actores institucionales internos tiene una marcada presencia. Este estilo aunque es positivo para dar a conocer el funcionamiento de la Institución, si es utilizado de forma redundante, puede incidir en que el actor se convierta en el tema central de la noticia, restándole importancia a la iniciativa o al objetivo que se quiere divulgar.

- La función del titular en la noticia reviste la mayor importancia, teniendo en cuenta, que la mayoría de periodistas filtran la información sólo con la información suministrada en este apartado. Por este motivo, es necesario dedicar tiempo a revisar cuestiones tales como si con el titular se capta la atención del público, y si se le puede considerar de tipo informativo.

- Es necesario establecer un formato definido (manual de estilo) que incluya cuestiones como: logotipo, tipo de letra, estructura y datos de contacto (teléfono fijo y móvil -con sus correspondientes extensionesy el correo electrónico). Esto ayuda a garantizar el refuerzo de la imagen institucional, y facilita la relación entre periodistas e institución.

\section{CONSIDERACIONES FINALES}

La imagen institucional pública de una determinada organización se consolida a través de las relaciones que genera con los medios de comunicación. Cada vez más, las instituciones son conscientes del valor relevante que significa que la información interna de las mismas, sea conocida por la opinión pública a través de los canales de información pública.

El conocimiento institucional a cargo de los responsables de comunicación en torno a la estrategia informativa para relacionarse con los medios, es determinante para el logro de los propios objetivos institucionales; sobre todo si se tiene en cuenta que los discursos mediados contribuyen a la creación, refuerzo y transformación de las representaciones sociales.

Mediaciones Sociales, № 9, II semestre 2011, pp. 111-133. ISSN electrónico: 1989-0494. 
La principal estrategia para reforzar esta relación empieza por definir con claridad un plan de comunicación donde las acciones con los medios estén establecidas y delimitadas bajo objetivos concretos. Los responsables de la ejecución de estas políticas de comunicación -por lo general los gabinetes de prensa-, deben coordinar una estructura de comunicación interna y externa coherente que permita representar de la mejor manera los objetivos institucionales de la organización.

Mientras que las labores de comunicación interna están encaminadas a identificar, establecer y mantener los objetivos de comunicación en el interior de la organización; las acciones de comunicación externa, deben reforzar la relación con los medios de comunicación por medio de estrategias de difusión de la actividad institucional, de seguimiento respecto a la calidad e incidencia informativa de sus mensajes, de la definición del tipo de públicos objetivos y del material informativo requerido para conseguir cada objetivo comunicativo planteado.

El recurso a las notas de prensa es el instrumento que propicia un primer acercamiento entre los productores de medios y el mensaje que a nivel institucional se quiere divulgar. Por este motivo, se hace necesario que en su realización, el comunicador tenga presente diferentes aspectos que pueden ser determinantes para conseguir captar los intereses de la agenda informativa de los medios.

Tanto las notas de prensa como otro material divulgativo que sirva de apoyo a la información suministrada, deben estar diseñados de acuerdo a los requerimientos propios de los medios informativos. Dichos requerimientos exigirán una aproximación al lenguaje informativo mediático, a la intencionalidad del mensaje, a la claridad y a los recursos útiles para responder a las demandas informativas de los medios. El objetivo de las notas debe ser lograr que la información institucional capte la atención de los encargados de decidir la agenda informativa.

Cada toma de contacto con los medios se debe aprovechar como una oportunidad única para generar nuevas oportunidades de intercambio comunicativo, donde la organización se posicione como una fuente de referencia útil para los periodistas sobre los temas básicos que le interesa hacer de conocimiento público.

Mediaciones Sociales, № 9, II semestre 2011, pp. 111-133. ISSN electrónico: 1989-0494. 


\section{Bibliografía}

Alcoceba, J. A. y Mathieu, G. (2008): Manual de buenas prácticas para el tratamiento de los jóvenes en los medios de comunicación. Madrid: Injuve. Disponible en http://www.injuve.es/contenidos.item.action? $\mathrm{id}=2017337609 \&$ menuId $=5720694$ $\underline{34}$ Consultado el 22 de julio de 2009.

Alcoceba Hernando, J. A. (2010): “Análisis de las notas de prensa institucionales y su visibilidad en la prensa”, Revista Latina de Comunicación Social, n 65, pp. 354-367. DOI: 10.4185/RLCS-65-2010-905-354-367; http://dx.doi.org/10.4185/RLCS-652010-905-354-367

Berger, P. y Luckman, T. (1966): La construcción social de la realidad. Buenos Aires: Amorrrortu.

Camacho Markina, I. (2001): “Comunicación institucional. Funciones de los gabinetes de comunicación en la administración local”, Revista Latina de Comunicación Social, $\mathrm{n}^{\circ} \quad 44 . \quad$ Disponible en http://www.ull.es/publicaciones/latina/2001/latina44septiembre/4403camac ho.htm. Consultado el 9 de diciembre de 2009.

Cancelo San Martín, M. M. (2007): “La comunicación gubernamental”, Chasqui, no 98 , pp. 72-75.

Canel, M. J. (2007): La Comunicación de las Instituciones Públicas. Madrid: Tecnos.

Coldwell, I. (2001): “The Ethics of Political Communication”, Political Studies Association Conference, Aberdeen, 7 de abril de 2001. Disponible en http://www.psa.ac.uk/journals/pdf/5/2002/coldwell.pdf. Consultado el 20 de septiembre de 2008.

Del Río Martínez, M. y otros (2001): Gabinetes de Prensa. La Comunicación en las Instituciones y en las empresas. Santander: Parlamento de Cantabria.

Domínguez Pérez, J. P. (2005): “El Poder en la Política de la Comunicación”, Revista Comunicologi@: indicios y conjeturas, $\mathrm{n}^{\circ} 4 . \quad$ Disponible en http://revistacomunicologia.org/index.php?option $=$ com_content\&task=view\&id $\equiv 106 \&$ Itemid $=76$. Consultado el 18 de mayo de 2009 .

García Rivas, M. (2003): "Presente de la información institucional de la Universidad española. El caso de la Universidad de Murcia”, Comunicación y Sociedad, vol. XVI, n ${ }^{\circ} 1$, pp. 29-56.

Mediaciones Sociales, № 9, II semestre 2011, pp. 111-133. ISSN electrónico: 1989-0494.

DOI: 10.5209/rev_MESO.2011.n9.38012 
Machado E Silva, E. (2004): "Representación de los jóvenes en el Discurso Político Institucional: Análisis de las Notas de Prensa Emitidas por la Secretaría General de Juventud de Catalunya - España”, XXVII Congresso Brasileiro de Ciências da Comunicação, Porto Alegre. Disponible en http://galaxy.intercom.org.br:8180/dspace/handle/1904/18017. Consultado el 23 de febrero de 2011.

Mateos Martín, C. (2003): "Descripción de la estructura funcional del gabinete de prensa político en los entornos informativos locales”, Revista Latina de Comunicación Social, $\mathrm{n}^{\circ} \quad 54$. Disponible en http://www.ull.es/publicaciones/latina/20035414mateos.htm. Consultado el 10 de enero de 2011 .

PÉREz CURIEL, C. (2008): "Comunicación política: un reto para la especialización de periodistas y fuentes”, Ámbitos, n” 17, pp. 251-269.

Ramos, F. (2002): “Comunicación e imagen corporativa”. Material de apoyo Curso de Publicidad, Temas I al IV, Universidad de Pontevedra, pp. 1-104. Disponible en http://www.slideshare.net/ferramos/comunicacin-e-imagen-corporativa-temas-ial-iv-4-curso-de-publicidad-pontevedra-profesor-dr-fernando-ramos. Consultado el 26 de noviembre de 2010

Revilla Castro, J. C. (2001): "La construcción discursiva de la juventud: lo general y lo particular”, Papers, n 63-64, pp. 103-122.

Santillán Buelna, J. R. (2010): "Political issue, un ejemplo de tematización periodística. Cómo los problemas públicos se seleccionan, discuten y solucionan en la prensa de referencia”, Congreso Internacional de la Asociación Española de Investigadores de la Comunicación (AE-IC) "Comunicación y desarrollo en la era digital", Málaga, 3-5 de febrero de 2010. Disponible en http://www.aeic2010malaga.org/upload/ok/220.pdf. Consultado el 12 de junio de 2009.

Xifra Triadú, J. (2008): "Modelos de las relaciones públicas políticas: Análisis de la situación en Cataluña”, Revista Latina de Comunicación Social, n 63, pp. 392-399. DOI: 10.4185/RLCS-63-2008-789-392-399; http://dx.doi.org/10.4185/RLCS-632008-789-392-399

VAn DiJK, T. A. (2004): Discurso y dominación. Bogotá: Universidad Nacional de Colombia. 


\section{PARA CITAR ESTE TRABAJO EN BIBLIOGRAFÍAS:}

ALCOCEBA HERNANDO, José Antonio y PINTO ARBOLEDA, María Cristina (2011): "Consideraciones y propuestas de mejora de la comunicación en las instituciones públicas”, Mediaciones Sociales. Revista de Ciencias Sociales y de la Comunicación, $\quad \mathrm{n}^{\circ} \quad$ 9, pp. 111-133. DOI: 10.5209/rev_MESO.2011.n9.38012; http://dx.doi.org/10.5209/rev_MESO.2011.n9.38012

\section{${ }^{(*)}$ Los autores}

José Antonio Alcoceba Hernando es licenciado y doctor en Ciencias de la Información por la Universidad Complutense de Madrid. Asistente Técnico del Instituto de la Juventud (Ministerio de Trabajo y Asuntos Sociales) desde el año 2000, ha realizado numerosas investigaciones en el campo de la juventud como investigador principal (II Encuesta a usuarios de servicios de información juvenil, Sondeos de Opinión de la Gente Joven, Coordinador Plan de Juventud 2005-2008). También ha elaborado diversos informes técnicos para la Unión Europea sobre Juventud y Voluntariado y Juventud e Información. Es coautor del libro Los Servicios de Información juvenil en España: un acercamiento a su realidad y funcionamiento, así como autor de diversos artículos y conferencias en el campo de la sociología de la juventud. Actualmente es Profesor Contratado Doctor en el Departamento de Sociología IV de la Facultad de Ciencias de la Información de la Universidad Complutense de Madrid.

María Cristina Pinto A. es comunicadora social y periodista egresada de la Universidad Externado de Colombia. Experta en Información Internacional y Países del Sur por la Universidad Complutense de Madrid. Actualmente realiza sus estudios de doctorado en Comunicación, Cambio Social y Desarrollo en el Departamento de Sociología IV de la Universidad Complutense de Madrid. Especializada en temas de comunicación para el desarrollo, ha desarrollado principalmente sus actividades profesionales en UNICEF (España y Nepal) y en el PNUD en Colombia.

RECIBIDO: 15 de septiembre de 2011.

ACEPTADO: 28 de octubre de 2011.

Mediaciones Sociales, № 9, II semestre 2011, pp. 111-133. ISSN electrónico: 1989-0494. DOI: 10.5209/rev_MESO.2011.n9.38012 
